\title{
Effect of Horizontal Heterogeneity on the Torsional Oscillation of an Elastic Sphere
}

\author{
By \\ Tatsuo USAMI \\ Earthquake Research Institute, the University of Tokyo
}

\section{§1. Introduction}

The three main factors affecting the free oscillation of the earth are the ellipticity, rotation and horizontal heterogeneity of the earth. The effect of the first two items have been studied by many seismologists [USAMI \& SATô (1962), PEKERIS et al. (1961), BACHUS and Gilbert (1961), CAputo (1963), MACDONALD and NESS (1961)] and the characteristics of the spectrum splitting associated with them have been made clear. SAITO (1971) has developed a general theory including the treatment of the effect of horizontal heterogeneity. In this study, the effect of horizontal heterogeneity on the period of free torsional oscillation and on the spectrum splitting is numerically investigated using variational method. The effect is generally less than $1 \%$ for long period oscillations.

\section{$\S 2$. Description of the theory}

The theory of variational approach to the general problem of the oscillation of an elastic sphere was developed in another paper [USAMI (1968)]. The result is summarized as follows.

Take a function $\phi$, which is the sum of strain energy function $(F)$ and kinetic energy $(K)$, namely,

$$
\phi=\frac{F}{2}+K
$$

and the integral $(I)$ over a spherical space

$$
I=\iiint \phi \cdot d v
$$

The condition that this integral takes a stationary value, or the variation of $(I)$ becomes null is equivalent to the equation of motion of an elastic sphere under the free surface conditions. In this theory, no assumption as to the vertical and/or horizontal heterogeneity is made. Harmonic oscillation is assumed. The strain energy function and kinetic energy have the expressions

$$
\left.\begin{array}{c}
K=-\frac{\rho p^{2}}{2}\left(U^{2}+V^{2}+W^{2}\right) \\
F=\overparen{r r} \cdot e_{r r}+\widehat{\theta \theta} \cdot e_{\theta \theta}+\widehat{\varphi \varphi} \cdot e_{\varphi \varphi}+\overparen{r \theta} \cdot e_{r \theta} \\
+\overparen{\theta \varphi} \cdot e_{\theta \varphi}+\widehat{\varphi r} \cdot e_{\varphi r}
\end{array}\right\}
$$

Therefore, $\phi$ is expressed as

$$
\begin{aligned}
\phi= & 2+2 \mu\left(U_{r}^{2}+V_{\theta}^{2}+W_{\varphi}^{2}\right) \\
& +\frac{\mu}{2}\left(U_{\theta}^{2}+U_{\varphi}^{2}+V_{r}^{2}+V_{\varphi}^{2}+W_{r}^{2}+W_{\theta}^{2}\right) \\
& +\lambda\left(U_{r} V_{\theta}+U_{r} W_{\varphi}+V_{\theta} W_{\varphi}\right) \\
& +\mu\left(U_{\theta} V_{r}+V_{\varphi} W_{r}+W_{\theta} V_{\varphi}\right) \\
& +\frac{\lambda}{r}(2 U+V \cdot \cot \theta)\left(U_{r}+V_{\theta}+W_{\varphi}\right) \\
& +\frac{2 \mu}{r}\left(U V_{\theta}+U W_{\varphi}+V W_{\varphi} \cot \theta\right) \\
& -\frac{\mu}{r}\left(V V_{r}+V U_{\theta}+W U_{\varphi}+W W_{r}\right) \\
& -\frac{\mu}{r} \cot \theta \cdot W\left(V_{\varphi}+W_{\theta}\right) \\
& +\frac{2(\lambda+\mu)}{r^{2}} U(U+V \cdot \cot \theta) \\
& +\frac{V^{2}}{2 r^{2}}\left(\mu+\overline{\lambda+2 \mu} \cdot \cot { }^{2} \theta\right) \\
& +\frac{\mu W^{2}}{2 r^{2}}\left(1+\cot { }^{2} \theta\right)-\frac{\rho p^{2}}{2}\left(U^{2}+V^{2}+W^{2}\right)
\end{aligned}
$$

where $U, V$ and $W$ mean the displacement component referred to the polar coordinates $(r, \theta, \varphi)$ and

$$
\begin{gathered}
\left\{\begin{array}{c}
U_{r} \\
V_{r} \\
W_{r}
\end{array}\right\}=\frac{\partial}{\partial r}\left\{\begin{array}{c}
U \\
V \\
W
\end{array}\right\}, \quad\left\{\begin{array}{c}
U_{\theta} \\
V_{\theta} \\
W_{\theta}
\end{array}\right\}=\frac{1}{r} \frac{\partial}{\partial \theta}\left\{\begin{array}{c}
U \\
V \\
W
\end{array}\right\}, \\
\left\{\begin{array}{c}
U_{\varphi} \\
V_{\varphi} \\
W_{\varphi}
\end{array}\right\}=\frac{1}{r \sin \theta} \frac{\partial}{\partial \varphi}\left\{\begin{array}{c}
U \\
V \\
W
\end{array}\right\}
\end{gathered}
$$


$\rho$ means the density and $p$ the frequency. is

The variation of the integral $I$ is

$$
\delta I=\iiint \delta \phi \cdot d v
$$

Since $\phi$ is a function of $U, V, W, U_{r}, \cdots W_{\theta}$ and $W_{\varphi}$, the variation of $\phi$ is calculated considering these quantities as independent variables.

\section{§3. Torsional oscillation-Simple example}

The word "standard state" is used to denote the state of no horizontal heterogeneity. In the standard state, only the torsional oscillation with axial symmetry $(m=0)$ is assumed. Generally speaking, even the standard state is pure torsional oscillation, additional spheroidal oscillation of relatively small amplitude will be generated by the horizontal heterogeneity. However, in the present case, $m=0$ is assumed in both the standard state and horizontal heterogeneity, the radial and colatitudinal displacements vanish always and no spheroidal oscillations appear. In the standard state, a homogeneous elastic sphere is adopted and, as to the heterogeneity, we put

$$
\begin{aligned}
& \mu=\mu_{0}\left\{1+\alpha(r) \cdot P_{s}(\cos \theta)\right\} \\
& \mu_{0}=\text { const }, \quad \alpha \ll 1
\end{aligned}
$$

where $\alpha(\gamma)$ is the coefficient defining the horizontal heterogeneity expressed by the spherical zonal harmonics. Although both the density and rigidity can be the cause of horizontal heterogeneity, the rigidity change is adopted. Because the change of density will require detailed consideration on the isostasic equilibrium which makes the problem more complicated. The Lame's constant $\lambda$ has nothing to do with the present problem.

The function $\phi$ has the form

$$
\begin{aligned}
\phi= & \frac{\mu}{2}\left(W_{r}^{2}+W_{\theta}{ }^{2}\right)-\frac{\mu}{r} W\left(W_{r}+\cot \theta \cdot W_{\theta}\right) \\
& +\frac{\mu W^{2}}{2 r^{2}}\left(1+\cot ^{2} \theta\right)-\frac{\rho}{2} p^{2} W^{2}
\end{aligned}
$$

This function does not involve the azimith $\varphi$. Therefore the condition that

$$
\delta I=0
$$

where

$$
\int_{0}^{a} r^{2} d r \int_{0}^{x} \sin \theta \cdot \delta \phi \cdot d \theta=0
$$

$$
\begin{aligned}
\delta \phi= & {\left[-\frac{\mu}{r}\left(W_{r}+\cot \theta \cdot W_{\theta}\right)\right.} \\
& \left.+\frac{2 \mu}{r^{2}} W\left(1+\cot ^{2} \theta\right)-\rho p^{2} W\right] \cdot \delta W \\
& +\mu\left(W_{r}-W / r\right) \frac{\partial}{\partial r}(\delta W) \\
& +\mu\left(W_{\theta}-\frac{W}{r} \cot \theta\right)_{r}^{1} \frac{\partial}{\partial \theta}(\delta W)
\end{aligned}
$$

The integral concerning the third line of $\delta \phi$ (eq. (11)) is

$$
\begin{gathered}
\int_{0}^{\pi} \sin \theta \cdot d \theta\left\{\left[\delta W \cdot \mu r^{2}\left(\frac{\partial W}{\partial r}-\frac{W}{r}\right)\right]_{r=0}^{a}\right. \\
\left.-\int_{0}^{a} \delta W \cdot \frac{\partial}{\partial r}\left[r^{2} \mu\left(\frac{\partial W}{\partial r}-\frac{W}{r}\right)\right] d r\right\} \\
=-\int_{0}^{\pi} \sin \theta \cdot d \theta \int_{0}^{a} \delta W \cdot \frac{\partial}{\partial r}\left(\mu r^{3} \frac{\partial}{\partial r}\left(\frac{W}{r}\right)\right) d r
\end{gathered}
$$

where the condition of free oscillation on the surface

$$
\frac{\partial W}{\partial r}-\frac{W}{r}=0
$$

is employed.

The integral related to the fourth line of $\delta \dot{\phi}$ is

$$
\begin{gathered}
\int_{0}^{a} d r \int_{0}^{\pi} \mu \sin \theta \cdot\left(\frac{\partial W}{\partial \theta}-W \cdot \cot \theta\right) \frac{\partial}{\partial \theta}(\delta W) d \theta \\
=\int_{0}^{a} d r\left\{\left[\delta W \cdot \mu \sin \theta\left(\frac{\partial W}{\partial \theta}-W \cot \theta\right)\right]_{\theta=0}^{\pi}\right. \\
\left.\quad-\int_{0}^{\pi} \delta W \cdot \frac{\partial}{\partial \theta}\left[\mu \sin \theta\left(\frac{\partial W}{\partial \theta}-W \cdot \cot \theta\right)\right] d \theta\right\} \\
=-\int_{0}^{a} d r \int_{0}^{\pi} \delta W \cdot \frac{\partial}{\partial \theta}[\mu \sin \theta \\
\left.\quad \times\left(\frac{\partial W}{\partial \theta}-W \cdot \cot \theta\right)\right] d \theta
\end{gathered}
$$

Finally, we have

$$
\begin{aligned}
\delta I= & \int_{0}^{a} d r \int_{0}^{\pi} \delta W\left[-\mu r \sin \theta \frac{\partial W}{\partial r}-\mu \cos \theta \frac{\partial W}{\partial \theta}\right. \\
& +\mu \sin \theta \cdot\left(1+\cot ^{2} \theta\right) W-\rho p^{2} r^{2} \sin \theta \cdot W
\end{aligned}
$$




$$
\begin{aligned}
& -\sin \theta \frac{\partial}{\partial r}\left(\mu r^{3} \frac{\partial}{\partial r}\left(\frac{W}{r}\right)\right) \\
& \left.-\frac{\partial}{\partial \theta}\left(\mu \sin \theta\left(\frac{\partial W}{\partial \theta}-\cot \theta \cdot W\right)\right)\right] d \theta=0
\end{aligned}
$$

In the standard state, the colatitudinal order number $n$ has a clear physical meaning, namely, the wave number measured near the equator. In a horizontally heterogeneous case, the number $N$, which corresponds to $n$ in the standard state, can have, when the horizontal heterogeneity is weak, approximate physiccal meaning. This situation is understandable, if we consider that, in a horizontally heterogeneous case, the phase velocity of surface wave (in the normal mode theory, each mode is interpreted as travelling along the surface) is different from place to place.

For the numerical work, only the radial fundamental mode is considered. It is assumed that

$$
W_{N}=\sum_{n=2}^{\infty} a_{n} W_{n}
$$

which means that the solution with order number $N$ can be expressed as a sum of solutions in the standard case with various order numbers $n$. Remember that the solutions $W_{0}$ and $W_{1}$ can not exist in the fundamental mode of standard case (SATô \& USAMI (1962)). It is expected that among the coefficients $a_{n}$, $a_{N}$ takes the maximum value so long as the horizontal heterogeneity is small. $W_{n}$ has the expression

$$
W_{n}=w_{n}(r) \cdot \frac{d}{d \theta} P_{n}(\cos \theta)
$$

and

$$
\left.\begin{array}{c}
\ddot{w}_{n}+\frac{2}{r} \dot{w}_{n}-\frac{n(n+1)}{r^{2}} w_{n}=-k_{n}{ }^{2} w_{n} \\
\ddot{P}_{n}+\cot \theta \cdot \dot{P}_{n}+n(n+1) P_{n}=0
\end{array}\right\}
$$

where $(\cdot)$ means $d / d r$ or $d / d \theta$ and $k_{n}=p_{n} / V_{s}$.

Putting (16) into (15), the integrand of the (15) becomes

$$
\begin{gathered}
\delta W \cdot \sum a_{n} I_{n} \\
I_{n}=-\sin \theta \cdot \dot{P}_{n} \frac{d}{d r}\left\{\mu r^{3} \frac{d}{d r}\left(\frac{w_{n}}{r}\right)\right\}
\end{gathered}
$$

$$
\begin{aligned}
& -w_{n}-\frac{d}{d \theta}\left\{\mu \sin \theta \cdot\left(\ddot{P}_{n}-\cot \theta \cdot \dot{P}_{n}\right)\right\} \\
& -\mu r \sin \theta \cdot \dot{w}_{n} \dot{P}_{n}-\mu \cos \theta \cdot w_{n} \cdot \ddot{P}_{n} \\
& -\rho p^{2} r^{2} w_{n} \cdot \sin \theta \cdot \dot{P}_{n} \\
& +\mu w_{n} \cdot \sin \theta \cdot\left(1+\cot ^{2} \theta\right) \cdot \dot{P}_{n}
\end{aligned}
$$

Employing the relations (7) and (18), $I_{n}$ is reduced to

$$
\begin{aligned}
I_{n}= & r^{2} w_{n} \sin \theta \cdot \dot{P}_{n}\left(\mu_{0} k_{n}{ }^{2}-\rho p^{2}\right) \\
& +\mu_{0} \alpha \sin \theta \cdot P_{s} \dot{P}_{n} r^{2} k_{n}{ }^{2} w_{n} \\
& +\mu_{0} \alpha \sin \theta \cdot \dot{P}_{s} w_{n}\left(2 \cot \theta \cdot \dot{P}_{n}+n(n+1) P_{n}\right) \\
& -\mu_{0} \dot{\alpha} \sin \theta \cdot P_{s} \dot{P}_{n} r^{2}\left(i \dot{v}_{n}-\frac{w_{n}}{r}\right)
\end{aligned}
$$

As the variation of $W_{N}$, we take

$$
\delta W_{N}=W_{n}, \quad\left(n^{\prime}=2,3, \cdots\right)
$$

Putting (16) and (22) into (21), the condition (15) requiring the zero-variation can be arranged in the form

$$
\sum_{n=2}^{n} a_{n} \cdot C_{n, n^{\prime}}=0 \quad\left(n^{\prime}=2,3, \cdots n\right)
$$

where

$$
\begin{gathered}
C_{n, n^{\prime}}=\frac{2 n^{\prime}\left(n^{\prime}+1\right)}{2 n^{\prime}+1} \delta \cdot\left(\mu_{0} k_{n^{\prime}}^{2}-\rho p^{2}\right) \int_{0}^{a} r^{2} w_{n^{\prime}}^{2} d r \\
+n(n+1) \mu_{0} \int_{0}^{\pi} \sin \theta \cdot \dot{P}_{s} P_{n} \dot{P}_{n^{\prime}} d \theta \int_{0}^{a} \alpha w_{n} w_{n^{\prime}} d r \\
+\mu_{0}{k_{n}}^{2} \int_{0}^{\pi} \sin \theta \cdot P_{s} \dot{P}_{n} \dot{P}_{n^{\prime}} d \theta \int_{0}^{a} \alpha r^{2} w_{n} w_{n^{\prime}} d r \\
+2 \mu_{0} \int_{0}^{\pi} \dot{P}_{s} \dot{P}_{n} \dot{P}_{n^{\prime}} \cdot \cos \theta d \theta \int_{0}^{a} \alpha w_{n} w_{n^{\prime}} d r \\
-\mu_{0} \int_{0}^{\pi} \sin \theta \cdot F_{s} \dot{P}_{n} \dot{P}_{n^{\prime}} d \theta \\
\times \int_{0}^{a} \dot{\alpha} r^{2} w_{n^{\prime}}\left(i_{n}-\frac{w_{n}}{r}\right) d r \\
\delta=\left\{\begin{array}{l}
0\left(n \neq n^{\prime}\right) \\
1\left(n=n^{\prime}\right)
\end{array}\right.
\end{gathered}
$$

The eq. (23) is the simultaneous equation with unknowns $a_{n}$. The upper limit of summation and of $n^{\prime}$ are put to $n$ for the convenience of numerical work. For the actual computation, the normalized radius $\zeta=r / a$ and radial wave number $k=p \sqrt{\rho / \mu_{0}}$ are introduced. In order that the eq. (23) has a non-zero solution, the determinant of the coefficients $C_{n, n}$ must be zero. From this condition, the non-dimensional 
frequency $\eta=k a$ will be calculated.

For the actual earth, the horizontal heterogeneity is reasonably considered to be confined in the shallow part, say, less than several hundred kilometers. Therefore, a discontinuity appears at the lower boundary of horizontally heterogeneous region. Effect of such a boundary is not involved in the formulas mentioned above. Let the radius of this boundary be $b$. Then, the effect of the discontinuity which appears in the first term of the expression (12) is

$$
\int_{0}^{\pi} \sin \theta \cdot d \theta\left[\delta W \cdot \mu r^{2}\left(\frac{\partial W}{\partial r}-\frac{W}{r}\right)\right]_{r=b+\varepsilon}^{r=b-\varepsilon}
$$

Employing expressions (16) and (7), this term becomes

$$
\begin{aligned}
& -\sum a_{n} \cdot \alpha b^{2}\left[w_{n^{\prime}} \cdot\left(\dot{w}_{n}-\frac{w_{n}}{r}\right)\right]_{r=b} \\
& \times \int_{0}^{\pi} \sin \theta \cdot P_{s} \dot{P}_{n} \dot{P}_{n^{\prime}} d \theta
\end{aligned}
$$

Therefore

$$
-\alpha b^{2}\left[w_{n^{\prime}} \cdot\left(\dot{w}_{n}-\frac{w_{n}}{r}\right)\right]_{r=b} \int_{0}^{\pi} \sin \theta \cdot P_{s} \dot{P}_{n} \dot{P}_{n^{\prime}} d \theta
$$

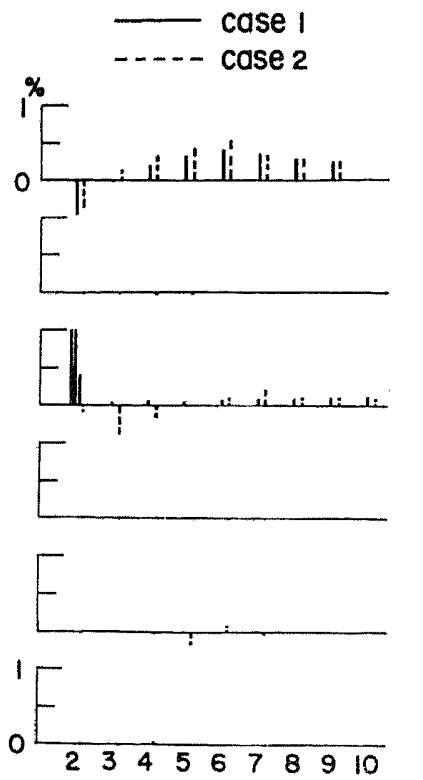

Fig. 1. Figure expressing $C_{r}$ in Table 1. Abscissa means $N$ in the eq. (16) of the text. must be added to $C_{n, n^{\prime}}$. This effect is considered in the case 2 of the next section.

\section{§4. Numerical Results}

\begin{tabular}{|c|c|c|c|}
\hline \multirow{2}{*}{ case } & \multicolumn{2}{|c|}{$\alpha$} & \multirow{2}{*}{ remark } \\
\hline & $\begin{array}{l}0.0<r \\
\leqq 0.9 a\end{array}$ & $\begin{array}{c}0.9 a<r \\
\leqq a\end{array}$ & \\
\hline 1 & 0.0 & 0.1 & \\
\hline 2 & 0.0 & 0.1 & $\begin{array}{l}\text { The effect of disconti- } \\
\text { nuity at the lower } \\
\text { boundary of the hetero- } \\
\text { geneous layer is } \\
\text { considered. }\end{array}$ \\
\hline 3 & 0.1 & 0.1 & \\
\hline
\end{tabular}

Numerical computations are carried out for the following three cases. $a$ in the table means the radius of the sphere.

The results are arranged in Table 1 and Fig. 1 , where $C_{r}=\left(\eta-\eta_{0}\right) / \eta_{0}$ is given. $\eta_{0}$ is the non-dimensional frequency [SATô and USAMI (1961)] of the standard state and $\eta$ is the corresponding quantity in the heterogeneous case. Theoretically, $\eta(=k a)$ has different value for different order of the determinant $\left|C_{n, n^{\prime}}\right|$. However, the discrepancy is negligibly small, suggesting the good accuracy of the adopted
$S=2$

3

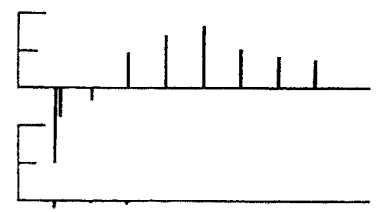

4

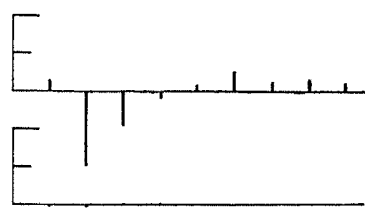

6
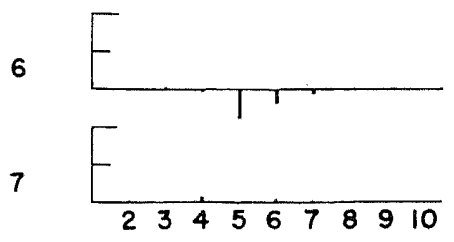
Table 1. Table of $C_{r}=\left(\eta-\eta_{0}\right) / \eta_{0} . \quad s$ is the colatitudinal order number of the horizontal heterogeneity expressed as the surface harmonics of degree $0 . N$ is the colatitudinal order number of the torsional oscillation. The upper, middle and lower numerals mean the cases 1,2 and 3 respectively.

\begin{tabular}{|c|c|c|c|c|c|c|c|c|c|}
\hline & 2 & 3 & 4 & 5 & 6 & 7 & 8 & 9 & 10 \\
\hline$s$ & $(2.501)$ & $(3.865)$ & $(5.095)$ & $(6.266)$ & $(7.404)$ & $(8.520)$ & $(9.621)$ & (10.711) & $(11.792)$ \\
\hline \multirow{3}{*}{2} & -0.0048 & -0.0001 & 0.0020 & 0.0033 & 0.0040 & 0.0035 & 0.0029 & 0.0025 & \\
\hline & -0.0038 & 0.0014 & 0.0031 & 0.0044 & 0.0051 & 0.0033 & 0.0029 & 0.0025 & \\
\hline & -0.0139 & -0.0016 & 0.0047 & 0.0070 & 0.0083 & 0.0051 & 0.0043 & 0.0037 & \\
\hline \multirow[t]{2}{*}{3} & -0.0001 & -0.0001 & -0.0002 & -0.0002 & & & & & \\
\hline & -0.0008 & -0.0002 & -0.0005 & $-0 .(4) 6$ & & & & & \\
\hline \multirow{3}{*}{4} & 0.0240 & 0.0002 & 0.0005 & 0.0005 & 0.0007 & 0.0009 & 0.0010 & 0.0011 & 0.0012 \\
\hline & -0.0007 & -0.0038 & -0.0016 & $0 .(4) 5$ & 0.0009 & 0.0021 & 0.0010 & 0.0011 & 0.0009 \\
\hline & 0.0014 & -0.0102 & -0.0047 & -0.0009 & 0.0007 & 0.0027 & 0.0013 & 0.0015 & 0.0009 \\
\hline \multirow[t]{2}{*}{5} & $-0 .(4) 8$ & -0.0001 & 0.0 & $0 .(4) 2$ & $-0 .(4) 1$ & $-0 .(4) 1$ & & & \\
\hline & -0.0003 & -0.0005 & $0 .(4) 6$ & 0.0001 & 0.0 & $-0 .(4) 4$ & & & \\
\hline \multirow[t]{2}{*}{6} & $-0 .(5) 2$ & $0 .(4) 3$ & -0.0002 & -0.0016 & 0.0007 & -0.0002 & & & \\
\hline & 0.0 & 0.0001 & -0.0003 & -0.0038 & -0.0018 & -0.0006 & & & \\
\hline \multirow[t]{2}{*}{7} & $0 .(4) 2$ & 0.0 & 0.0003 & $-0 .(4) 2$ & & & & & \\
\hline & $0 .(4) 2$ & 0.0 & 0.0006 & -0.0001 & 0.0003 & $0 .(4) 3$ & & & \\
\hline
\end{tabular}

method. From the Table, the following conclusions are obtained.

1) The effect of horizontal heterogeneity on the free torsional oscillation frequency is small, being less than $1 \%$, even though the assumed heterogeneity is large compared to that estimated for the actual earth.

2) $C_{r}$ changes its sign periodically as a function of the colatitudinal order number $N$. The peak amplitude diminishes as $N$ increases.

3) The effect of discontinuity becomes small as $N$ increases. This effect can not be neglected for very long period oscillation.

4) Generally $C_{r}$ decrease, but not monotonously, as the order of horizontal heterogeneity $s$ in the expression (7) increases.

5) Contrary to the expectation, $C_{r}$ does not necessarily take the maximum value when $N$ is equal to $s$.
6) The numerical work was done for relatively long period oscillations. We might imagine that the effect of horizontal heterogeneity has practically negligible value even for short period oscillation of actual earth.

7) Since the azimuthal order number $m$ is assumed to be zero in both the standard state and the horizontal heterogeneity, the spectrum splitting did not appear in the case adopted here.

The author wishes to express his sincere thanks to the Computer Centre, the University of Tokyo who courteously permitted him to use the HITAC 5020E.

\section{References}

Backus, G. and F. Gilbert, The rotational splitting of the free oscillation of the earth, Proc. Nat. Acad. Sci., 47, 362-371, 1961. 
Caputo, M., Free modes of layered oblate planets, J.G.R., 68, 497-503, 1963.

MacDonald, G. J.F. and N. F. Ness, A study of the free oscillations of the earth, J. Geophys. Res., 66, 1865-1912, 1961.

Pekeris, K. L., Z. Alterman and H. Jarosch, Comparison of theoretical with observed values of the periods of free oscillations of the earth, Proc. Nat. Acad. Sci., 47, 91-98, 1961.

Saito, M., Theory for the elastic-gravitational oscillation of a laterally heterogeneous earth, $J$. Phys. Earth, 19, No. 3, 1971 (in press).
Satô, Y. and T. Usami, Basic study on the oscillation of a homogeneous elastic sphere, I Frequency of the free oscillations, Geophys. Mag., 31, $15-24,1962$.

Usami, T., Expression of variational method for the oscillation problem of an elastic sphere, (in Japanese), Zisin, ii, 21, 143-146, 1968.

Usami, T., and Y. Satô, Torsional oscillations of a homogeneous elastic spheroid, Bull. Seism. Soc. Amer., 52, 469-484, 1962.

(Received on April 27, 1971) 